NTUA-59/96

IFUNAM-FT $97-4$

hep-ph/9704218

\title{
Reduction of Couplings and Finiteness in Realistic Supersymmetric GUTs
}

\author{
J. Kubo \\ Physics Dept. \\ Faculty of Natural Sciences \\ Kanazawa University \\ 920-11 Kanazawa, Japan \\ M. Mondragón柿 \\ Instituto de Física, UNAM \\ Apdo. Postal 20-364 \\ México 1000 D.F., México \\ and \\ G. Zoupanos \\ Physics Deptartment \\ Nat. Technical University \\ 15780 Zografou, Athens, Greece
}

\begin{abstract}
Reduction of couplings in supersymmetric GUTs is achieved by searching for renormalization group invariant (RGI) relations among couplings which hold beyond the unification scale. Finiteness is due to the fact that there exist RGI relations among couplings that guarantee the vanishing of the $\beta$ functions of a $N=1$ supersymmetric GUT even to all orders in perturbation theory. Of particular interest are the relations among gauge and Yukawa couplings which lead to very interesting predictions of the top quark mass.
\end{abstract}

\footnotetext{
*Presented by G. Zoupanos at the Buckow Symposium 1996

${ }^{\dagger}$ Partially supported by the PAPIIT project IN110296

${ }^{\ddagger}$ Partially supported by the E.C. projects CHRX-CT93-0319, ERBFMRXCT960090, and the Greek project PENED/1170
} 


\section{Introduction}

The essence of all theoretical efforts in Elementary Particle Physics (EPP) is to understand the present day free parameters of the Standard Model (SM) in terms of a few fundamental ones, i.e. to achieve reduction of couplings. The pathology of the plethora of free parameters is well known. It is deeply connected to the presence of infinities at the quantum level. The renormalization program can remove the infinities by introducing counterterms, but only at the cost of leaving the corresponding terms as free parameters. The traditional way of reducing the number of parameters is to assume that the world is more symmetric at higher scales. A celebrated example along this line is the minimal $S U(5)$, whose gauge sector provided us with a testable prediction for one of the low energy gauge couplings. In fact nowadays LEP data suggest that the minimal $S U(5)$ GUT should be even more symmetric, specifically $N=1$ supersymmetric. However, requiring more symmetry, e.g. constructing GUTs based on groups of higher rank, such as $S O(10), E_{6}, E_{7}, E_{8}$, is well known that does not increase the predictive power of these models. An extreme case from this point of view are superstrings or superbranes, which have huge symmetries but no predictions for the SM parameters.

A natural extension of the GUT idea is to find a way to relate the gauge and Yukawa sectors of a theory, that is to achieve Gauge-Yukawa Unification (GYU). A symmetry which naturally relates the two sectors is supersymmetry, in particular $N=2$ supersymmetry. It turns out, however, that $N=2$ supersymmetric theories have serious phenomenological problems due to light mirror fermions. Also, in superstring theories and in composite models there exist relations among the gauge and Yukawa couplings, but both kind of theories have phenomenological problems.

In a series of papers [1, 2, 3, 4, 33 we have proposed another way to relate the gauge and Yukawa sectors of a theory. It is based on the fact that within the framework of a renormalizable field theory, one can find renormalization group invariant (RGI) relations among parameters that can improve the calculability and the predictive power of a theory. We have considered models in which the GYU is achieved using the principles of reduction of couplings [6, 7, 8] and finiteness [1], [12]- 21], 25]-[28], [32]. These principles, which are formulated in perturbation theory, are not explicit symmetry principles, although they might imply symmetries [11]. The reduction of couplings is based on the existence of RGI relations among couplings, which preserve 
perturbative renormalizability.

The principle of finiteness maybe today requires some more motivation in order to be generally accepted. It is however interesting to note that in the old days the general feeling was quite different. Probably the well known Dirac's phrase that "...divergences are hidden under the carpet" is representative of the views of that time.

In recent years we have a more relaxed attitude towards divergencies. Most theorists believe that the divergencies are signals of the existence of a higher scale, where new degrees of freedom are excited. Parenthetically one should note that even accepting this philosophy everybody is bothered by the presence of quadratic divergencies in a theory, since their presence implies that physics at one scale is very sensitive to unknown physics at higher scales. This was and still remains the main reason for introducing supersymmetric theories in EPP, since they provide us with the only known perturbative examples of theories free of quadratic divergences.

Returning to the argument that divergences reflect the existence of new physics at higher scales, we are naturally led to the conclusion that beyond unification scale, i.e. when all interactions have been taken into account in a unified scheme, the theory should be completely finite. In fact this is one of the main motivations and aims of string, non-commutative geometry and quantum group theories, which include also gravity in the unification of the interactions.

In our attempts here we are restricted in unifying only the known gauge interactions, and it is interesting to point out that finiteness does not require gravity. Finiteness is based on the fact that it is possible to find RGI relations among couplings that keep finiteness in perturbation theory, even to all orders.

Applying the principles of reduction of couplings and finiteness one can relate the gauge and Yukawa couplings and therefore improve the predictive power of a model.

In the following we present the principles of reduction of couplings and finiteness as well as their application in realistic supersymmetric GUTs. In addition we present some hints on the deeper connections among the reduction of couplings, supersymmetry and finiteness which certainly requires further exploration. 


\section{Reduction of Couplings by the RGI Method}

Let us next briefly outline the idea of reduction of couplings. Any RGI relation among couplings (which does not depend on the renormalization scale $\mu$ explicitly) can be expressed, in the implicit form $\Phi\left(g_{1}, \cdots, g_{A}\right)=$ const., which has to satisfy the partial differential equation (PDE)

$$
\mu \frac{d \Phi}{d \mu}=\vec{\nabla} \cdot \vec{\beta}=\sum_{a=1}^{A} \beta_{a} \frac{\partial \Phi}{\partial g_{a}}=0,
$$

where $\beta_{a}$ is the $\beta$-function of $g_{a}$. This PDE is equivalent to a set of ordinary differential equations, the so-called reduction equations (REs) [7],

$$
\beta_{g} \frac{d g_{a}}{d g}=\beta_{a}, a=1, \cdots, A,
$$

where $g$ and $\beta_{g}$ are the primary coupling and its $\beta$-function, and the counting on $a$ does not include $g$. Since maximally $(A-1)$ independent RGI "constraints" in the $A$-dimensional space of couplings can be imposed by the $\Phi_{a}$ 's, one could in principle express all the couplings in terms of a single coupling $g$. The strongest requirement is to demand power series solutions to the REs,

$$
g_{a}=\sum_{n=0} \rho_{a}^{(n)} g^{2 n+1}
$$

which formally preserve perturbative renormalizability. Remarkably, the uniqueness of such power series solutions can be decided already at the oneloop level [7]. To illustrate this, let us assume that the $\beta$-functions have the form

$$
\begin{aligned}
\beta_{a} & =\frac{1}{16 \pi^{2}}\left\{\sum_{b, c, d \neq g} \beta_{a}^{(1) b c d} g_{b} g_{c} g_{d}\right. \\
& \left.+\sum_{b \neq g} \beta_{a}^{(1) b} g_{b} g^{2}\right\}+\cdots, \\
\beta_{g} & =\frac{1}{16 \pi^{2}} \beta_{g}^{(1)} g^{3}+\cdots,
\end{aligned}
$$

where $\cdots$ stands for higher order terms, and $\beta_{a}^{(1) b c d}$ 's are symmetric in $b, c, d$. We then assume that the $\rho_{a}^{(n)}$ 's with $n \leq r$ have been uniquely determined. 
To obtain $\rho_{a}^{(r+1)}$ 's, we insert the power series (3) into the REs (2) and collect terms of $O\left(g^{2 r+3}\right)$ to find

$$
\sum_{d \neq g} M(r)_{a}^{d} \rho_{d}^{(r+1)}=\text { lower order quantities }
$$

where the r.h.s. is known by assumption, and

$$
\begin{aligned}
M(r)_{a}^{d} & =3 \sum_{b, c \neq g} \beta_{a}^{(1) b c d} \rho_{b}^{(1)} \rho_{c}^{(1)}+\beta_{a}^{(1) d} \\
& -(2 r+1) \beta_{g}^{(1)} \delta_{a}^{d} \\
0 & =\sum_{b, c, d \neq g} \beta_{a}^{(1) b c d} \rho_{b}^{(1)} \rho_{c}^{(1)} \rho_{d}^{(1)} \\
& +\sum_{d \neq g} \beta_{a}^{(1) d} \rho_{d}^{(1)}-\beta_{g}^{(1)} \rho_{a}^{(1)}
\end{aligned}
$$

Therefore, the $\rho_{a}^{(n)}$ 's for all $n>1$ for a given set of $\rho_{a}^{(1)}$ 's can be uniquely determined if $\operatorname{det} M(n)_{a}^{d} \neq 0$ for all $n \geq 0$.

It is clear by examining specific examples, that the various couplings in supersymmetric theories have easily the same asymptotic behaviour. Therefore searching for a power series solution of the form (3) to the REs (21) is justified. This is not the case in non-supersymmetric theories.

The above facts lead us to suspect that there is and intimate connection among the requirement of reduction of couplings and supersymmetry which still waits to be uncovered. The connection becomes more clear by examining the following example.

Consider an $S U(N)$ gauge theory with the following matter content: $\phi^{i}(\mathbf{N})$ and $\hat{\phi}_{i}(\overline{\mathbf{N}})$ are complex scalars, $\psi^{i}(\mathbf{N})$ and $\hat{\psi}_{i}(\overline{\mathbf{N}})$ are left-handed Weyl spinor, and $\lambda^{a}\left(a=1, \ldots, N^{2}-1\right)$ is right-handed Weyl spinor in the adjoint representation of $S U(N)$.

The Lagrangian is:

$$
\begin{aligned}
& \mathcal{L}=-\frac{1}{4} F_{\mu \nu}^{a} F^{a \mu \nu}+i \sqrt{2}\left\{g_{Y} \bar{\psi} \lambda^{a} T^{a} \phi\right. \\
& \left.-\hat{g}_{Y} \overline{\hat{\psi}} \lambda^{a} T^{a} \hat{\phi}+\text { h.c. }\right\}-V(\phi, \bar{\phi}), \\
& V(\phi, \bar{\phi})=\frac{1}{4} \lambda_{1}\left(\phi^{i} \phi_{i}^{*}\right)^{2}+\frac{1}{4} \lambda_{2}\left(\hat{\phi}_{i} \hat{\phi}^{* i}\right)^{2} \\
& +\lambda_{3}\left(\phi^{i} \phi_{i}^{*}\right)\left(\hat{\phi}_{j} \hat{\phi}^{* j}\right)+\lambda_{4}\left(\phi^{i} \phi_{j}^{*}\right)\left(\hat{\phi}_{i} \hat{\phi}^{* j}\right),
\end{aligned}
$$


which is the most general renormalizable form of dimension four, consistent with the $S U(N) \times S U(N)$ global symmetry.

Searching for solution (3) of the REs (2) we find in lowest order the following one:

$$
\begin{aligned}
& g_{Y}=\hat{g}_{Y}=g, \\
& \lambda_{1}=\lambda_{2}=\frac{N-1}{N} g^{2}, \\
& \lambda_{3}=\frac{1}{2 N} g^{2}, \lambda_{4}=-\frac{1}{2} g^{2} .
\end{aligned}
$$

which corresponds to an $N=1$ supersymmetric gauge theory 1 . Clearly the above remarks do not answer the question of the relation among reduction of couplings and supersymmetry but rather try to trigger the interest for further investigation.

\section{Reduction of Couplings and $\mathrm{N}=1$ Super- symmetric Gauge Theories}

Let us consider a chiral, anomaly free, $N=1$ globally supersymmetric gauge theory based on a group $\mathrm{G}$ with gauge coupling constant $g$. The superpotential of the theory is given by

$$
W=\frac{1}{2} m_{i j} \phi_{i} \phi_{j}+\frac{1}{6} C_{i j k} \phi_{i} \phi_{j} \phi_{k},
$$

where $m_{i j}$ and $C_{i j k}$ are gauge invariant tensors and the matter field $\phi_{i}$ transforms according to the irreducible representation $R_{i}$ of the gauge group $G$.

The $N=1$ non-renormalization theorem [24] ensures that there are no mass and cubic-interaction-term infinities. As a result the only surviving possible infinities are the wave-function renormalization constants $Z_{i}^{j}$, i.e., one infinity for each field. The one -loop $\beta$-function of the gauge coupling $g$ is given by 12

$$
\beta_{g}^{(1)}=\frac{d g}{d t}=\frac{g^{3}}{16 \pi^{2}}\left\{\sum_{i} l\left(R_{i}\right)-3 C_{2}(G)\right\},
$$

\footnotetext{
${ }^{1}$ Further details will be given in a future publication [34].
} 
where $l\left(R_{i}\right)$ is the Dynkin index of $R_{i}$ and $C_{2}(G)$ is the quadratic Casimir of the adjoint representation of the gauge group $G$. The $\beta$-functions of $C_{i j k}$, by virtue of the non-renormalization theorem, are related to the anomalous dimension matrix $\gamma_{i j}$ of the matter fields $\phi_{i}$ as:

$$
\beta_{i j k}=\frac{d C_{i j k}}{d t}=C_{i j l} \gamma_{k}^{l}+C_{i k l} \gamma_{j}^{l}+C_{j k l} \gamma_{i}^{l}
$$

At one-loop level $\gamma_{i j}$ is 12

$$
\gamma_{i j}^{(1)}=\frac{1}{32 \pi^{2}}\left\{C^{i k l} C_{j k l}-2 g^{2} C_{2}\left(R_{i}\right) \delta_{i j}\right\}
$$

where $C_{2}\left(R_{i}\right)$ is the quadratic Casimir of the representation $R_{i}$, and $C^{i j k}=$ $C_{i j k}^{*}$. Since dimensional coupling parameters such as masses and couplings of cubic scalar field terms do not influence the asymptotic properties of a theory on which we are interested here, it is sufficient to take into account only the dimensionless supersymmetric couplings such as $g$ and $C_{i j k}$. So we neglect the existence of dimensional parameters, and assume furthermore that $C_{i j k}$ are real so that $C_{i j k}^{2}$ always are positive numbers. For our purposes, it is convenient to work with the square of the couplings and to arrange $C_{i j k}$ in such a way that they are covered by a single index $i(i=1, \cdots, n)$ :

$$
\alpha=\frac{|g|^{2}}{4 \pi}, \alpha_{i}=\frac{\left|g_{i}\right|^{2}}{4 \pi} \text {. }
$$

We define

$$
\tilde{\alpha}_{i} \equiv \frac{\alpha_{i}}{\alpha}, i=1, \cdots, n,
$$

and derive a form for the evolution equations in terms of $\alpha$ and $\tilde{\alpha}$ :

$$
\begin{gathered}
\alpha \frac{d \tilde{\alpha}_{i}}{d \alpha}=-\tilde{\alpha}_{i}+\frac{\beta_{i}}{\beta}=\left(-1+\frac{\beta_{i}^{(1)}}{\beta^{(1)}}\right) \tilde{\alpha}_{i} \\
-\sum_{j, k} \frac{\beta_{i, j k}^{(1)}}{\beta^{(1)}} \tilde{\alpha}_{j} \tilde{\alpha}_{k}+\sum_{r=2}\left(\frac{\alpha}{\pi}\right)^{r-1} \tilde{\beta}_{i}^{(r)}(\tilde{\alpha}),
\end{gathered}
$$

where $\tilde{\beta}_{i}^{(r)}(\tilde{\alpha})(r=2, \cdots)$ are power series of $\tilde{\alpha}$ 's and can be computed from the $r$-th loop $\beta$-functions. Next we search for fixed points $\rho_{i}$ of Eq. (15) at 
$\alpha=0$. To this end, we have to solve

$$
\left(-1+\frac{\beta_{i}^{(1)}}{\beta^{(1)}}\right) \rho_{i}-\sum_{j, k} \frac{\beta_{i, j k}^{(1)}}{\beta^{(1)}} \rho_{j} \rho_{k}=0,
$$

and assume that the fixed points have the form

$$
\begin{aligned}
\rho_{i}= & 0 \text { for } i=1, \cdots, n^{\prime} ; \\
& \rho_{i}>0 \text { for } i=n^{\prime}+1, \cdots, n .
\end{aligned}
$$

We then regard $\tilde{\alpha}_{i}$ with $i \leq n^{\prime}$ as small perturbations to the undisturbed system which is defined by setting $\tilde{\alpha}_{i}$ with $i \leq n^{\prime}$ equal to zero. As we have seen, it is possible to verify at the one-loop level [7] the existence of the unique power series solution

$$
\begin{array}{r}
\tilde{\alpha}_{i}=\rho_{i}+\sum_{r=2} \rho_{i}^{(r)} \alpha^{r-1}, \\
i=n^{\prime}+1, \cdots, n
\end{array}
$$

of the reduction equations (16) to all orders in the undisturbed system. These are RGI relations among couplings and keep formally perturbative renormalizability of the undisturbed system. So in the undisturbed system there is only one independent coupling, the primary coupling $\alpha$.

\subsection{The Minimal Supersymmetric $S U(5)$ Model}

The minimal $\mathrm{N}=1$ supersymmetric $S U(5)$ model is particularly interesting, being the the simplest GUT supported by the LEP data. Here we will consider it as an attractive example of a partially reduced model. Its particle content is well defined and has the following transformation properties under SU(5): three $(\overline{\mathbf{5}}+\mathbf{1 0})$ - supermultiplets which accommodate three fermion families, one $(\mathbf{5}+\overline{\mathbf{5}})$ to describe the two Higgs supermultiplets appropriate for electroweak symmetry breaking and a $\mathbf{2 4}$-supermultiplet required to provide the spontaneous symmetry breaking of $S U(5)$ down to $S U(3) \times S U(2) \times U(1)$.

We require the GYU to occur among the Yukawa couplings of the third generation and the gauge coupling. In addition we require the theory to be

completely asimptotically free. 
Table 1: The predictions of the minimal SUSY SU(5)

\begin{tabular}{|c|c|c|c|c|c|c|c|}
\hline$m_{\mathrm{SUSY}}[\mathrm{GeV}]$ & $g_{t}^{2} / g^{2}$ & $g_{b}^{2} / g^{2}$ & $\alpha_{3}\left(M_{Z}\right)$ & $\tan \beta$ & $M_{\mathrm{GUT}}[\mathrm{GeV}]$ & $m_{b}[\mathrm{GeV}]$ & $m_{t}[\mathrm{GeV}]$ \\
\hline 300 & 0.97 & 0.57 & 0.120 & 47.7 & $1.8 \times 10^{16}$ & 5.4 & 179.7 \\
\hline 500 & 0.97 & 0.57 & 0.118 & 47.7 & $1.39 \times 10^{16}$ & 5.3 & 178.9 \\
\hline
\end{tabular}

Neglecting the dimensional parameters and the Yukawa couplings of the first two generations, the superpotential of the model is exactly given by

$$
\begin{aligned}
W= & \frac{1}{2} g_{t} \mathbf{1 0}_{3} \mathbf{1 0}_{3} H+\sqrt{2} g_{b} \overline{\mathbf{5}}_{3} \mathbf{1 0}_{3} \bar{H} \\
& +\frac{1}{3} g_{\lambda}(\mathbf{2 4})^{3}+g_{f} \bar{H} \mathbf{2 4} H,
\end{aligned}
$$

where $H, \bar{H}$ are the $\mathbf{5}, \overline{\mathbf{5}}$ - Higgs supermultiplets and we have suppressed the $S U(5)$ indices.

In the one-loop approximation, the GYU yields

$$
g_{t, b}^{2}=\sum_{m, n=1}^{\infty} \kappa_{t, b}^{(m, n)}\left[\frac{g_{\lambda}}{g}\right]^{2 m}\left[\frac{g_{f}}{g}\right]^{2 n} g^{2}
$$

( $h$ and $f$ are related to the Higgs couplings). Where $\left[g_{\lambda} / g\right]^{2}$ is allowed to vary from 0 to $15 / 7$, while $\left[g_{f} / g\right]^{2}$ may vary from 0 to a maximum which depends on $\left[g_{\lambda} / g\right]^{2}$ and vanishes at $\left[g_{\lambda} / g\right]^{2}=15 / 7$. As a result, we obtain [5]

$$
\begin{gathered}
0.97 g^{2} \leq g_{t}^{2} \leq 1.37 g^{2}, \\
0.57 g^{2} \leq g_{b}^{2} \quad=g_{\tau}^{2} \leq 0.97 g^{2} .
\end{gathered}
$$

We found [5] that consistency with proton decay requires $g_{t}^{2}, g_{b}^{2}$ to be very close to the left hand side values in the inequalities.

Just below the unification scale we would like to obtain the MSSM $S U(3) \times$ $S U(2) \times U(1)$ and one pair of Higgs doublets, and assume that all the superpartners are degenerate at the supersymmetry breaking scale, where the MSSM will be broken to the normal SM. Then the standard model should 
be spontaneously broken down to $S U(3) \times U(1)_{\mathrm{em}}$ due to the VEVs of the two Higgs $S U(2)$-doublets contained in the $\mathbf{5}, \overline{\mathbf{5}}$-super-multiplets.

One way to obtain the correct low energy theory is to add to the Lagrangian soft supersymmetry breaking terms and to arrange the mass parameters in the superpotential along with the soft breaking terms so that the desired symmetry breaking pattern of the original $S U(5)$ is really the preferred one, all the superpartners are unobservable at present energies, there is no contradiction with proton decay, and so forth. Then we study the evolution of the couplings at two loops respecting all the boundary conditions at $M_{G U T}$.

In Table 1 we give the predictions for representative values of $m_{S U S Y}$, where we have suppressed the threshold effects of the superheavy as well as of the MSSM particles.

\section{Finiteness and Reduction of Couplings in Extended Supersymmetric Gauge Theories}

In the introduction we have presented arguments suggesting that a truly unified theory should be finite. The two main new points that we would like to advertise in the present and the following two chapters are a) that there exist finite gauge theories, i.e. unified theories which are finite without the inclusion of gravity, and b) that there exists a finite realistic GUT with testable low energy predictions. Furthermore we would like to present suggestive hints on the deeper relation among reduction of couplings and finiteness which reopens the old relevant discussion.

Concerning finiteness in gauge theories it is well known that there exists a non-renormalization theorem [9], which guarantees that gauge theories based on any gauge group with extended $N=4$ supersymmetry is finite to all orders. On the other hand it is also well known that nobody even dares to attempt the construction of realistic models based on $N=4$.

The next best candidates gauge theories to become finite are those having $N=2$ supersymmetry. The reason is the existence of another nonrenormalization theorem [10] stating that their $\beta$-functions obtain only oneloop contributions. Therefore for a given gauge group one could choose, in principle, appropriate matter fields so as to obtain a vanishing one-loop $\beta$ - 
function and then the non-renormalization theorem guarantees the finiteness to all orders. To be more specific, the $\beta$-function of a $N=2$ gauge theory is [114]:

$$
\beta(g)=\frac{2 g^{3}}{4 \pi^{2}}\left(\sum_{i} T\left(R_{i}\right)-C_{2}(G)\right) .
$$

Therefore a $N=2$ gauge theory based on the group $S U(N)$ could be made finite by introducing $2 N$ multiplets transforming according to the fundamental representation. In particular searching for finite $N=2$ GUTs one finds [15] that the introduction of the matter fields in the following representations and corresponding multiplicities is required:

$$
\mathbf{S U}(\mathbf{5}): \quad p(\mathbf{5}+\overline{\mathbf{5}}) ; \quad q(\mathbf{1 0}+\overline{\mathbf{1 0}}) ; r(\mathbf{1 5}+\overline{\mathbf{1 5}})
$$

with the multiplicities $p, q, r$ satisfying the constraint

$$
\begin{gathered}
p+3 q+7 r=10 . \\
\mathbf{S O}(\mathbf{1 0}): \quad p(\mathbf{1 0}+\overline{\mathbf{1 0}}) ; \quad q(\mathbf{1 6}+\overline{\mathbf{1 6}})
\end{gathered}
$$

with

$$
\begin{gathered}
p+2 q=8 . \\
\left.\mathbf{E}_{(} \mathbf{6}\right): \quad 4(\mathbf{2 7}+\overline{\mathbf{2 7}})
\end{gathered}
$$

Obviously the above GUTs, in order to become finite, require the presence of multiplets corresponding to mirror particles.

Therefore, the construction of a realistic finite GUT necessitates the invention of a mechanism which keeps ordinary fermions light and makes the mirror fermions unobservably heavy. Due to the lack of such a mechanism at present, the question of constructing realistic $N=2$ GUTs is postponed but certainly not excluded.

Let us turn next to a discussion of the relation among extended supersymmetries and the reduction of couplings by examining specific gauge theories with the particle content of $N=2,4$ pure super Yang-Mills (SYM) theories (i.e. without matter fields) but without the corresponding couplings [11, [16]. 


\subsection{Frame theory for $N=2$ SYM}

Consider the following $R$-invariant Lagrangian

$$
\begin{aligned}
L & =-\frac{1}{4 g^{2}} F^{\mu \nu} F_{\mu \nu}+\frac{1}{2}\left(D_{\mu} \phi^{a}\right)^{2}+\frac{1}{2}\left(D_{\mu} \pi^{a}\right)^{2} \\
& -i \bar{\psi}^{a} \gamma D \psi^{a}-i \sqrt{g_{1}} \epsilon^{a b c} \bar{\psi}\left(\phi^{a}+\gamma_{5} \pi^{b}\right) \psi^{c} \\
& -\frac{1}{4} g_{2}\left(\phi^{2}+\pi^{2}\right)^{2}+\frac{1}{4} g_{3}\left(\phi^{a} \phi^{b}+\pi^{a} \pi^{b}\right)^{2},
\end{aligned}
$$

where we have a supermultiplet $\phi^{a}$ of real scalar, $\pi^{a}$ of pseudoscalar, $A_{\mu}^{a}$ of vector field, and $\psi^{a}$ of Dirac spinor, all transforming according to the adjoint representation of $S U(2)$. The Lagrangian has the field content of pure $N=2$ SYM-theory in components and allows for a non-supersymmetric embedding of it. Searching for power series solutions (3) of the REs (2) one finds two solutions of the RE with positive classical potential, and uniquely determined at all orders. One which has the $N=2$ symmetry at tree level

$$
\rho_{1}^{(0)}=\rho_{2}^{(0)}=\rho_{3}^{(0)}=1,
$$

and one which does not have the symmetry

$$
\rho_{1}^{(0)}=1, \quad \rho_{2}^{(0)}=\frac{9}{\sqrt{105}}, \quad \rho_{3}^{(0)}=\frac{7}{\sqrt{105}} .
$$

\subsection{Frame Theory for $N=4$ SYM}

Consider now the following $N=4$ SYM Lagrangian, which is required to be $S U(2) \times S U(2)$ rigid invariant for simplicity

$$
\begin{aligned}
L & =-\frac{1}{4 g^{2}} F^{\mu \nu} F_{\mu \nu}+\frac{1}{2}\left(D_{\mu} \phi_{i}^{a}\right)^{2} \\
& +\frac{1}{2}\left(D_{\mu} \pi_{l}^{a}\right)^{2}-\frac{i}{2} \bar{\psi}_{K}^{a} \not D \psi_{K}^{a} \\
& -\frac{1}{2} \sqrt{g_{1}} \epsilon^{a b c} \bar{\psi}_{K}^{a}\left(\alpha_{K L}^{i} \phi_{i}^{b}+\gamma_{5} \beta_{K L}^{i} \pi_{i}^{b}\right) \psi_{L}^{c} \\
& -\frac{1}{4} g_{2}\left(\phi_{i}^{a} \phi_{i}^{a}+\pi_{l}^{a} \pi_{l}^{a}\right)^{2}+\frac{1}{4} g_{2}\left(\phi_{i}^{a} \phi_{i}^{b}+\pi_{l}^{a} \pi_{l}^{b}\right)^{2},
\end{aligned}
$$

where we have 3 scalar $\phi_{i}, 3$ pseudoscalars $\pi_{i}, 4$ Majorana spinors $\psi_{K}$, and one vector $A_{\mu}$, all transforming according to the adjoint representation of $S U(2)$. 
Searching again for solutions of the RE (21) we get two allowed power series solutions (3). The first one has $N=4$ symmetry

$$
\rho_{1}^{(0)}=\rho_{2}^{(0)}=\rho_{3}^{(0)}=1
$$

The second one does not show any symmetry at the tree approximation

$$
\rho_{1}^{(0)}=1, \quad \rho_{2}^{(0)}=0.7579 \ldots, \quad \rho_{3}^{(0)}=0.2523 \ldots
$$

and it is worth noticing that its $\beta$-functions vanish in the one-loop order also.

Thus we see that the requirement of reduction of couplings admits a symmetric solution corresponding to the extended supersymmetric theories but it is more general, since it has also other solutions.

\section{$5 \quad$ Finiteness and Reduction of Couplings in $N=1$ SUSY Gauge Theories}

As one can see from Eqs. (11) and (13) in Chapter 3, all the one-loop $\beta$ functions of the theory vanish if $\beta_{g}^{(1)}$ and $\gamma_{i j}^{(1)}$ vanish, i.e.

$$
\begin{gathered}
\sum_{i} \ell\left(R_{i}\right)=3 C_{2}(G), \\
C^{i k l} C_{j k l}=2 \delta_{j}^{i} g^{2} C_{2}\left(R_{i}\right),
\end{gathered}
$$

A very interesting result is that the conditions (34, 35) are necessary and sufficient for finiteness at the two-loop level [12].

In case supersymmetry is broken by soft terms, one-loop finiteness of the soft sector imposes further constraints on it [17]. In addition, the same set of conditions that are sufficient for one-loop finiteness of the soft breaking terms render the soft sector of the theory two-loop finite [18.

The one- and two-loop finiteness conditions (34,35) restrict considerably the possible choices of the irreps. $R_{i}$ for a given group $G$ as well as the Yukawa couplings in the superpotential (10). Note in particular that the finiteness conditions cannot be applied to the supersymmetric standard model (SSM), since the presence of a $U(1)$ gauge group is incompatible with the condition (34), due to $C_{2}[U(1)]=0$. This naturally leads to the expectation that 
finiteness should be attained at the grand unified level only, the SSM being just the corresponding, low-energy, effective theory.

Another important consequence of one- and two-loop finiteness is that supersymmetry (most probably) can only be broken by soft breaking terms. Indeed, due to the unacceptability of gauge singlets, F-type spontaneous symmetry breaking 22 terms are incompatible with finiteness, as well as Dtype 23] spontaneous breaking which requires the existence of a $U(1)$ gauge group.

A natural question to ask is what happens at higher loop orders. The answer is contained in a theorem [25] which states the necessary and sufficient conditions to achieve finiteness at all orders. Before we discuss the theorem let us make some introductory remarks. The finiteness conditions impose relations between gauge and Yukawa couplings. To require such relations which render the couplings mutually dependent at a given renormalization point is trivial. What is not trivial is to guarantee that relations leading to a reduction of the couplings hold at any renormalization point. As we have seen, the necessary, but also sufficient, condition for this to happen is to require that such relations are solutions to the REs

$$
\beta_{g} \frac{d C_{i j k}}{d g}=\beta_{i j k}
$$

and hold at all orders. As we have seen, remarkably the existence of all-order power series solutions to (36) can be decided at the one-loop level.

Let us now turn to the all-order finiteness theorem [25], which states when a $N=1$ supersymmetric gauge theory can become finite to all orders in the sense of vanishing $\beta$-functions, that is of physical scale invariance. It is based on (a) the structure of the supercurrent in $N=1 \mathrm{SYM}$ [30, 31], and on (b) the non-renormalization properties of $N=1$ chiral anomalies [25, 26]. Details on the proof can be found in refs. 25 and further discussion in refs. [26, 27, 28]. Here, following mostly ref. [28] we present a comprehensible sketch of the proof.

Consider a $N=1$ supersymmetric gauge theory, with simple Lie group $G$. The content of this theory is given at the classical level by the matter supermultiplets $S_{i}$, which contain a scalar field $\phi_{i}$ and a Weyl spinor $\psi_{i a}$, and the vector supermultiplet $V_{a}$, which contains a gauge vector field $A_{\mu}^{a}$ and a gaugino Weyl spinor $\lambda_{\alpha}^{a}$.

Let us first recall certain facts about the theory: 
(1) A massless $N=1$ supersymmetric theory is invariant under a $U(1)$ chiral transformation $R$ under which the various fields transform as follows

$$
\begin{aligned}
A_{\mu}^{\prime} & =A_{\mu}, \quad \lambda_{\alpha}^{\prime}=\exp (-i \theta) \lambda_{\alpha} \\
\phi^{\prime} & =\exp \left(-i \frac{2}{3} \theta\right) \phi, \quad \psi_{\alpha}^{\prime}=\exp \left(-i \frac{1}{3} \theta\right) \psi_{\alpha}, \cdots
\end{aligned}
$$

The corresponding axial Noether current $J_{R}^{\mu}(x)$ is

$$
J_{R}^{\mu}(x)=\bar{\lambda} \gamma^{\mu} \gamma^{5} \lambda+\cdots
$$

is conserved classically, while in the quantum case is violated by the axial anomaly

$$
\partial_{\mu} J_{R}^{\mu}=r\left(\epsilon^{\mu \nu \sigma \rho} F_{\mu \nu} F_{\sigma \rho}+\cdots\right) .
$$

From its known topological origin in ordinary gauge theories [29], one would expect that the axial vector current $J_{R}^{\mu}$ to satisfy the Adler-Bardeen theorem and receive corrections only at the one-loop level. Indeed it has been shown that the same non-renormalization theorem holds also in supersymmetric theories [26]. Therefore

$$
r=\hbar \beta_{g}^{(1)} .
$$

(2) The massless theory we consider is scale invariant at the classical level and, in general, there is a scale anomaly due to radiative corrections. The scale anomaly appears in the trace of the energy momentum tensor $T_{\mu \nu}$, which is traceless classically. It has the form

$$
T_{\mu}^{\mu}=\beta_{g} F^{\mu \nu} F_{\mu \nu}+\cdots
$$

(3) Massless, $N=1$ supersymmetric gauge theories are classically invariant under the supersymmetric extension of the conformal group - the superconformal group. Examining the superconformal algebra, it can be seen that the subset of superconformal transformations consisting of translations, supersymmetry transformations, and axial $R$ transformations is closed under supersymmetry, i.e. these transformations form a representation of supersymmetry. It follows that the conserved currents corresponding to these transformations make up a supermultiplet represented by an axial vector superfield called supercurrent $J$,

$$
J \equiv\left\{J_{R}^{\prime \mu}, Q_{\alpha}^{\mu}, T_{\nu}^{\mu}, \ldots\right\}
$$


where $J_{R}^{\prime \mu}$ is the current associated to $\mathrm{R}$ invariance, $Q_{\alpha}^{\mu}$ is the one associated to supersymmetry invariance, and $T_{\nu}^{\mu}$ the one associated to translational invariance (energy-momentum tensor).

The anomalies of the $\mathrm{R}$ current $J_{R}^{\prime \mu}$, the trace anomalies of the supersymmetry current, and the energy-momentum tensor, form also a second supermultiplet, called the supertrace anomaly

$$
\begin{aligned}
S= & \left\{\operatorname{Re} S, \operatorname{Im} S, S_{\alpha}\right\}= \\
& \left\{T_{\mu}^{\mu}, \partial_{\mu} J_{R}^{\prime \mu}, \sigma_{\alpha \dot{\beta}}^{\mu} \bar{Q}_{\mu}^{\dot{\beta}}+\cdots\right\}
\end{aligned}
$$

where $T_{\mu}^{\mu}$ in Eq.(41) and

$$
\begin{aligned}
\partial_{\mu} J_{R}^{\prime \mu} & =\beta_{g} \epsilon^{\mu \nu \sigma \rho} F_{\mu \nu} F_{\sigma \rho}+\cdots \\
\sigma_{\alpha \dot{\beta}}^{\mu} \bar{Q}_{\mu}^{\dot{\beta}} & =\beta_{g} \lambda^{\beta} \sigma_{\alpha \beta}^{\mu \nu} F_{\mu \nu}+\cdots
\end{aligned}
$$

(4) It is very important to note that the Noether current defined in (38) is not the same as the current associated to $R$ invariance that appears in the supercurrent $J$ in (42), but they coincide in the tree approximation. So starting from a unique classical Noether current $J_{R(\text { class })}^{\mu}$, the Noether current $J_{R}^{\mu}$ is defined as the quantum extension of $J_{R \text { (class) }}^{\mu}$ which allows for the validity of the non-renormalization theorem. On the other hand $J_{R}^{\prime \mu}$, is defined to belong to the supercurrent $J$, together with the energy-momentum tensor. The two requirements cannot be fulfilled by a single current operator at the same time.

Although the Noether current $J_{R}^{\mu}$ which obeys (39) and the current $J_{R}^{\prime \mu}$ belonging to the supercurrent multiplet $J$ are not the same, there is a relation [25] between quantities associated with them

$$
r=\beta_{g}\left(1+x_{g}\right)+\beta_{i j k} x^{i j k}-\gamma_{A} r^{A}
$$

where $r$ was given in Eq. (40). The $r^{A}$ are the non-renormalized coefficients of the anomalies of the Noether currents associated to the chiral invariances of the superpotential, and -like $r$ - are strictly one-loop quantities. The $\gamma_{A}$ 's are linear combinations of the anomalous dimensions of the matter fields, and $x_{g}$, and $x^{i j k}$ are radiative correction quantities. The structure of equality (46) is independent of the renormalization scheme.

One-loop finiteness, i.e. vanishing of the $\beta$-functions at one-loop, implies that the Yukawa couplings $\lambda_{i j k}$ must be functions of the gauge coupling $g$. 
To find a similar condition to all orders it is necessary and sufficient for the Yukawa couplings to be a formal power series in $g$, which is solution of the REs (36).

We can now state the theorem for all-order vanishing $\beta$-functions.

\section{Theorem:}

Consider an $N=1$ supersymmetric Yang-Mills theory, with simple gauge group. If the following conditions are satisfied

1. There is no gauge anomaly.

2. The gauge $\beta$-function vanishes at one-loop

$$
\beta_{g}^{(1)}=0=\sum_{i} l\left(R_{i}\right)-3 C_{2}(G)
$$

3. There exist solutions of the form

$$
C_{i j k}=\rho_{i j k} g, \quad \rho_{i j k} \in \mathbb{C}
$$

to the conditions of vanishing one-loop matter fields anomalous dimensions

$$
\begin{aligned}
& \gamma_{j}^{i(1)}=0 \\
& =\frac{1}{32 \pi^{2}}\left[C^{i k l} C_{j k l}-2 g^{2} C_{2}\left(R_{i}\right) \delta_{i j}\right] .
\end{aligned}
$$

4. these solutions are isolated and non-degenerate when considered as solutions of vanishing one-loop Yukawa $\beta$-functions:

$$
\beta_{i j k}=0
$$

Then, each of the solutions (48) can be uniquely extended to a formal power series in $g$, and the associated super Yang-Mills models depend on the single coupling constant $g$ with a $\beta$ function which vanishes at all-orders.

It is important to note a few things: The requirement of isolated and nondegenerate solutions guarantees the existence of a unique formal power series

solution to the reduction equations. The vanishing of the gauge $\beta$-function 
Table 2: The predictions of the FUT $S U(5)$

\begin{tabular}{|c|c|c|c|c|c|}
\hline$m_{\mathrm{SUSY}}[\mathrm{GeV}]$ & $\alpha_{3}\left(M_{Z}\right)$ & $\tan \beta$ & $M_{\mathrm{GUT}}[\mathrm{GeV}]$ & $m_{b}[\mathrm{GeV}]$ & $m_{t}[\mathrm{GeV}]$ \\
\hline 200 & 0.123 & 53.7 & $2.25 \times 10^{16}$ & 5.2 & 184.0 \\
\hline 500 & 0.118 & 54.2 & $1.45 \times 10^{16}$ & 5.1 & 184.4 \\
\hline
\end{tabular}

at one-loop, $\beta_{g}^{(1)}$, is equivalent to the vanishing of the $\mathrm{R}$ current anomaly (39). The vanishing of the anomalous dimensions at one-loop implies the vanishing of the Yukawa couplings $\beta$-functions at that order. It also implies the vanishing of the chiral anomaly coefficients $r^{A}$. This last property is a necessary condition for having $\beta$ functions vanishing at all orders 2 .

\section{Proof:}

Insert $\beta_{i j k}$ as given by the REs into the relationship (46) between the axial anomalies coefficients and the $\beta$-functions. Since these chiral anomalies vanish, we get for $\beta_{g}$ an homogeneous equation of the form

$$
0=\beta_{g}(1+O(\hbar))
$$

The solution of this equation in the sense of a formal power series in $\hbar$ is $\beta_{g}=0$, order by order. Therefore, due to the REs (36), $\beta_{i j k}=0$ too.

Thus we see that finiteness and reduction of couplings are intimately related.

\section{Finite SU(5) from reduction of couplings}

Let us next consider a realistic Finite Unified Model based on $S U(5)$. From the classification of theories with vanishing one-loop $\beta$ function for the gauge coupling [13], one can see that using $S U(5)$ as gauge group there exist only two candidate models which can accommodate three fermion generations. These models contain the chiral supermutiplets $\mathbf{5}, \overline{\mathbf{5}}, \mathbf{1 0}, \overline{\mathbf{5}}, \mathbf{2 4}$ with the multiplicities $(6,9,4,1,0)$ and $(4,7,3,0,1)$, respectively. Only the second one contains a 24-plet which can be used for spontaneous symmetry breaking

\footnotetext{
${ }^{2}$ There is an alternative way to find finite theories 19 .
} 
(SSB) of $S U(5)$ down to $S U(3) \times S U(2) \times U(1)$. (For the first model one has to incorporate another way, such as the Wilson flux breaking to achieve the desired SSB of $S U(5)$ [1]). Therefore, we would like to concentrate only on the second model.

To simplify the situation, we neglect the intergenerational mixing among the lepton and quark supermultiplets and consider the following $S U(5)$ invariant cubic superpotential for the (second) model:

$$
\begin{aligned}
& W= \sum_{i=1}^{3} \sum_{\alpha=1}^{4}\left[\frac{1}{2} g_{i \alpha}^{u} \mathbf{1 0}_{i} \mathbf{1 0}_{i} H_{\alpha}\right. \\
&\left.+g_{i \alpha}^{d} \mathbf{1 0}_{i} \overline{\mathbf{5}}_{i} \bar{H}_{\alpha}\right] \\
&+\sum_{\alpha=1}^{4} g_{\alpha}^{f} H_{\alpha} \mathbf{2 4} \bar{H}_{\alpha}+\frac{g^{\lambda}}{3}(\mathbf{2 4})^{3}, \\
& \text { with } g_{i \alpha}^{u, d}=0 \text { for } i \neq \alpha,
\end{aligned}
$$

where the $\mathbf{1 0}_{i}$ 's and $\overline{\mathbf{5}}_{i}$ 's are the usual three generations, and the four $(\mathbf{5}+$ $\overline{5})$ Higgses are denoted by $H_{\alpha}, \bar{H}_{\alpha}$. The superpotential is not the most general one, but by virtue of the non-renormalization theorem, this does not contradict the philosophy of the coupling unification by the reduction method (a RG invariant fine tuning is a solution of the reduction equation). In the case at hand, however, one can find a discrete symmetry $Z_{3} \times Z_{7}$, together with a multiplicative $Q$-parity, that can be imposed on the most general cubic superpotential to arrive at the non-intergenerational mixing [1].

It is very interesting that demanding reduction of couplings we find a unique power series solution (3) of the dimensionless parameters of the theory in favour of the gauge coupling $g$. The unique power series solution [1] corresponds to the Yukawa matrices without intergenerational mixing, and yields in the one-loop approximation

$$
\begin{aligned}
g_{t}^{2}=g_{c}^{2} & =g_{u}^{2}=\frac{8}{5} g^{2}, \\
g_{b}^{2}=g_{s}^{2}=g_{d}^{2} & =\frac{6}{5} g^{2}, \\
g_{\tau}^{2}=g_{\mu}^{2} & =g_{e}^{2}=\frac{6}{5} g^{2},
\end{aligned}
$$

where $g_{i}$ 's stand for the Yukawa couplings. 
Moreover, the above unique solution gives in lowest order vanishing matter fields anomalous dimensions $\gamma_{j}^{i(1)}$, and Yukawa $\beta$-functions $\beta_{i j k}$. Therefore the conditions of the $N=1$ finiteness theorem of Chapter 5 are satisfied which in turns guarantees the finiteness of the theory to all orders in perturbation theory. The point that we would like to stress here is that we have chosen a theory with $\beta_{g}^{(1)}=0$ with a superpotential restricted by some symmetries, and we have demanded reduction of couplings. We did not impose $\gamma_{j}^{i(1)}=0$. This came out as a bonus of the requirement of reduction of couplings 3 and shows an intimate connection among the reduction of couplings and finiteness in a class of supersymmetric models with $\beta_{g}^{(1)}=0$.

At first sight, this GYU seems to lead to unacceptable predictions of the fermion masses. But this is not the case, because each generation has an own pair of $(\overline{\mathbf{5}}+\mathbf{5})$-Higgses so that one may assume [20, 1] that after the diagonalization of the Higgs fields the effective theory is exactly MSSM, where the pair of its Higgs supermultiplets mainly stems from the $(\mathbf{5}+\overline{\mathbf{5}})$ which couples to the third fermion generation. (The Yukawa couplings of the first two generations can be regarded as free parameters.) The predictions of $M_{t}$ and $M_{b}$ for various $M_{\text {SUSY }}$ are given in Table 2, where we have suppressed the threshold effects of the superheavy as well as of the MSSM superparticles.

Adding soft breaking terms (which are supposed not to influence the $\beta$ functions beyond $M_{\mathrm{GUT}}$ ), we can obtain supersymmetry breaking. The conditions on the soft breaking terms to preserve one and two-loop finiteness have been given in refs. [17, 18], as we have already mentioned. It is an open problem whether there exists a suitable set of conditions on the soft terms for all-loop finiteness.

\section{Conclusions}

As a natural extension of the unification of gauge couplings provided by all GUTs and the unification of Yukawa couplings, we have introduced the idea of Gauge-Yukawa Unification. GYU is a functional relationship among the gauge and Yukawa couplings provided by some principle. In our studies GYU has been achieved by applying the principles of reduction of couplings and finiteness. The consequence of GYU is that in the lowest order in perturbation theory the gauge and Yukawa couplings above $M_{\mathrm{GUT}}$ are related in the

\footnotetext{
${ }^{3}$ Further details will be given in a future publication 34 .
} 


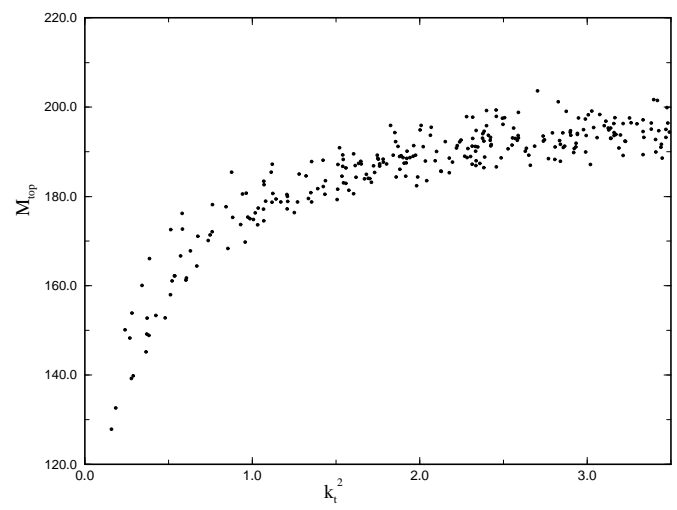

Figure 1: The dependence of the top mass $M_{t}$ with $k_{t}^{2}$, at fixed $M_{S U S Y}=500$ $\mathrm{GeV}$. As we can see, after $k_{t}^{2} \sim 2.0$ the top mass goes to its infrared fixed point value.

form

$$
g_{i}=\kappa_{i} g_{\mathrm{GUT}}, i=1,2,3, e, \cdots, \tau, b, t,
$$

where $g_{i}(i=1, \cdots, t)$ stand for the gauge and Yukawa couplings, $g_{\text {GUT }}$ is the unified coupling, and we have neglected the Cabibbo-Kobayashi-Maskawa mixing of the quarks. So, Eq. (56) exhibits a set of boundary conditions on the the renormalization group evolution for the effective theory below $M_{\mathrm{GUT}}$, which we have assumed to be the MSSM. We have shown [5] that it is possible to construct some supersymmetric GUTs with GYU in the third generation that can predict the bottom and top quark masses in accordance with the recent experimental data. This means that the top-bottom hierarchy could be explained in these models, in a similar way as the hierarchy of the gauge couplings of the SM can be explained if one assumes the existence of a unifying gauge symmetry at $M_{\mathrm{GUT}}$.

It is clear that the GYU scenario is the most predictive scheme as far as the mass of the top quark is concerned. It may be worth recalling the predictions for $M_{t}$ of ordinary GUTs, in particular of supersymmetric $S U(5)$ and $S O(10)$. The MSSM with $S U(5)$ Yukawa boundary unification allows $M_{t}$ to be anywhere in the interval between $100-200 \mathrm{GeV}$ for varying $\tan \beta$, which is now a free parameter. Similarly, the MSSM with $S O(10)$ Yukawa 
boundary conditions, i.e. $t-b-\tau$ Yukawa Unification, gives $M_{t}$ in the interval 160-200 GeV. We have analyzed [5] the infrared quasi-fixed-point behaviour of the $M_{t}$ prediction in some detail. In particular we have seen that the infrared value for large $\tan \beta$ depends on $\tan \beta$ and its lowest value is $\sim 188 \mathrm{GeV}$. Comparing this with the experimental value $m_{t}=(176.8 \pm 6.5)$ $\mathrm{GeV}$ we may conclude that the present data on $M_{t}$ cannot be explained from the infrared quasi-fixed-point behaviour alone (see Figure 1).

Clearly, to exclude or verify different GYU models, the experimental as well as theoretical uncertainties have to be further reduced. One of the largest theoretical uncertainties in FUT results from the not-yet-calculated threshold effects of the superheavy particles. Since the structure of the superheavy particles is basically fixed, it will be possible to bring these threshold effects under control, which will reduce the uncertainty of the $M_{t}$ prediction [5]. There we have been regarding the MSSM threshold correction $\delta^{\mathrm{MSSM}} M_{t}$ as unknown because we do not have sufficient information on the superpartner

spectra. Recently, however, we have demonstrated [33] how to extend the principle of reduction of couplings in a way as to include the dimensionfull parameters. As a result, it is in principle possible to predict the superpartner spectra as well as the rest of the massive parameters of a theory.

\section{Acknowledgements}

It is a pleasure for one of us (G.Z) to thank the Organizing Committee for the warm hospitality.

\section{References}

[1] D. Kapetanakis, M. Mondragón and G. Zoupanos, Zeit. f. Phys. C60 (1993) 181; M. Mondragón and G. Zoupanos, Nucl. Phys. B (Proc. Suppl) 37C (1995) 98.

[2] J. Kubo, M. Mondragón and G. Zoupanos, Nucl. Phys. B424 (1994) 291.

[3] J. Kubo, M. Mondragón, N.D. Tracas and G. Zoupanos, Phys. Lett. B342 (1995) 155. 
[4] J. Kubo, M. Mondragón, S. Shoda and G. Zoupanos, Nucl. Phys. B469 (1996) 3.

[5] J. Kubo, M. Mondragón, M. Olechowski and G. Zoupanos, Nucl. Phys. B479 (1996) 25.

[6] T.P. Cheng, E. Eichten and L.F. Li, Phys. Rev. D9 (1974) 2259; N.P. Chang, Phys. Rev. D10 (1974) 2706; E. Ma, Phys. Rev D17 (1978) 623; ibid D31 (1985) 1143.

[7] W. Zimmermann, Com. Math. Phys. 97 (1985) 211;147 R. Oehme and W. Zimmermann Com. Math. Phys. 97 (1985) 569; R. Oehme, K. Sibold and W. Zimmermann, Phys. Lett. B147 (1984) 117; B153 (1985) 142 .

[8] J. Kubo, K. Sibold and W. Zimmermann, Nucl. Phys. B259 (1985) 331; Phys. Lett. B200 (1989) 185.

[9] M. Sonius and P. West, Phys. Lett B100 (1981) 45; S. Ferrara and B. Zumino, unpublished.

[10] M. Grisaru and W. Siegel, Nucl. Phys. B201 (1982) 292.

[11] K. Sibold, Acta Phys. Pol. 19b (1988) 295.

[12] A.J. Parkes and P.C. West, Phys. Lett. B138 (1984) 99; Nucl. Phys. B256 (1985) 340; P. West, Phys. Lett. B137 (1984) 371; D.R.T. Jones and A.J. Parkes, Phys. Lett. B160 (1985) 267; D.R.T. Jones and L. Mezinescu, Phys. Lett. B136 (1984) 242; B138 (1984) 293; A.J. Parkes, Phys. Lett. B156 (1985) 73.

[13] S. Hamidi, J. Patera and J.H. Schwarz, Phys. Lett. B141 (1984) 349; X.D. Jiang and X.J. Zhou, Phys. Lett. B197 (1987) 156; B216 (1985) 160.

[14] S. Ferrara and B. Zumino, Nucl. Phys. B79 (1974) 413.

[15] A. Parkes and P. West, Phys. Lett. B127 (1983) 248.

[16] R. Oehme, K. Sibold and W. Zimmermann, Phys. Lett. B147 (1984) 115. 
[17] D.R.T. Jones, L. Mezincescu and Y.-P. Yao, Phys. Lett. B148 (1984) 317.

[18] I. Jack and D.R.T. Jones, Phys.Lett. B333 (1994) 372.

[19] R.G. Leigh and M.J. Strassler, Nucl. Phys. B447 (1995) 95.

[20] S. Hamidi and J.H. Schwarz, Phys. Lett. B147 (1984) 301; D.R.T. Jones and S. Raby, Phys. Lett. B143 (1984) 137; J.E. Bjorkman, D.R.T. Jones and S. Raby Nucl. Phys. B259 (1985) 503.

[21] J. León et al, Phys. Lett. B156 (1985) 66.

[22] L. O'Raifeartaigh, Nucl. Phys. B96 (1975) 331.

[23] P. Fayet and J. Iliopoulos, Phys. Lett. B51 (1974) 461.

[24] J. Wess and B. Zumino, Phys. Phys. B49 52; J. Iliopoulos and B. Zumino, Nucl. Phys. B76 (1974) 310; S. Ferrara, J. Iliopoulos and B. Zumino, Nucl. Phys. B77 (1974) 413; K. Fujikawa and W. Lang, Nucl. Phys. B88 (1975) 61.

[25] C. Lucchesi, O. Piguet and K. Sibold, Helv. Phys. Acta 61 (1988) 321; Phys. Lett. B201 (1988) 241.

[26] O. Piguet and K. Sibold, Int. Journ. Mod. Phys. A1 (1986) 913; Phys. Lett. B177 (1986) 373; R. Ensign and K.T. Mahanthappa, Phys. Rev. D36 (1987) 3148.

[27] C. Lucchesi and G. Zoupanos, hep-ph/9604216.

[28] O. Piguet, hep-th/9606045.

[29] L. Alvarez-Gaumé and P. Ginsparg, Nucl. Phys. B243 (1984) 449; W.A. Bardeen and B. Zumino, Nucl. Phys.B243 (1984) 421; B. Zumino, Y. Wu and A. Zee, Nucl. Phys. B439 (1984) 477.

[30] S. Ferrara and B. Zumino, Nucl. Phys. B87 (1975) 207.

[31] O. Piguet and K. Sibold, Nucl. Phys. B196 (1982) 428; B196 (1982) 447. 
[32] A.V. Ermushev, D.I. Kazakov and O.V. Tarasov, Nucl. Phys. B281 (1987) 72; D.I. Kazakov, Mod. Phys. Let. A2 (1987) 663; Phys. Lett. B179 (1986) 352.

[33] J. Kubo, M. Mondragón and G. Zoupanos, hep-ph/9609218. To be published in Phys. Lett. B.

[34] J. Kubo, M. Mondragón and G. Zoupanos, in preparation. 